Assessment of dermal water by high-frequency ultrasound: comparative studies with nuclear magnetic resonance.

Quistorff, Bjørn; Gniadecka, M.

Published in:

British Journal of Dermatology

Publication date:

1996

Document version

Publisher's PDF, also known as Version of record

Citation for published version (APA):

Quistorff, B., \& Gniadecka, M. (1996). Assessment of dermal water by high-frequency ultrasound: comparative studies with nuclear magnetic resonance. British Journal of Dermatology, 135(2), 218-224. 


\title{
Assessment of dermal water by high-frequency ultrasound: comparative studies with nuclear magnetic resonance
}

\author{
M.GNIADECKA AND B.QUISTORFF* \\ Bioengineering and Skin Research Laboratory, Department of Dermatology, University of Copenhagen, Bispebjerg Hospital, Denmark \\ *Nuclear Magnetic Resonance Centre, Panum Institute, University of Copenhagen, Denmark \\ Accepted for publication 18 December 1995
}

Summary Although a principal constituent of human skin, cutaneous water is difficult to study, and little is known about water behaviour in physiological and pathological conditions of the skin. It has been proposed recently that changes in dermal echogenicity measured by high-frequency ultrasonography reflect changes in dermal water content. To validate skin ultrasonography for assessment of dermal water changes we have studied the relationship between dermal echogenicity and skin water content determined by nuclear magnetic resonance technique. Twenty $\mathrm{MHz}$ ultrasound scanning of the dorsal and ventral forearm skin was performed in 59 healthy volunteers (age 18-65) and dermal echogenicity was determined by counting low echogenic pixels (LEPs) in skin images. ${ }^{1} \mathrm{H}$ magnetic resonance spectra were obtained from the same regions and the ratio of areas under the water- and fat-specific peaks $(\mathrm{W} / \mathrm{F})$ were calculated to measure a relative water content. Acute dermal oedema (histamine weal) was studied in the same way in 40 individuals. Baseline dermal echogenicity correlated significantly with W/F, both in the ventral $(r=0.47)$ and dorsal $(r=0.57)$ forearm. Intradermal application of histamine caused a development of intradermal oedema which could be visualized by nuclear magnetic resonance imaging. In a corresponding ultrasound image oedema was seen as a low-echogenic area. The proportional increases in LEPs and W/F after histamine application were correlated, but the elevation in LEPs was $25-48 \%$ (95\% confidence intervals) higher than that for $\mathrm{W} / \mathrm{F}$. These results suggest that high-frequency ultrasonography is a sensitive method for assessment of changes in dermal hydration. This technique may find important applications in comparative and non-invasive evaluations of dermal water in physiological conditions and in skin pathologies associated with oedema formation.

Water constitutes about $70 \%$ of the mass of human skin, provides adequate environment for metabolic processes, and to a great extent determines skin structure, turgor and other physical properties. Despite being so abundant, water is a very difficult component to study. The assessment of the changes in tissue volume due to the influx of water is insensitive as swelling is only apparent when the volume of interstitial fluid is doubled. ${ }^{1,2}$ Invasive skin sampling procedures involving skin biopsies and histological processing invariably cause alterations in water distribution and content.

High resolution $20 \mathrm{MHz}$ ultrasonography is a noninvasive procedure which has been increasingly used for studying cutaneous water. ${ }^{3-11}$ In the dermis,

Correspondence: Monika Gniadecka, MD, PhD, Frederiksberg Allé 29. 3. t.v. 1820 Frederiksberg C, Denmark. ultrasound reflections are generated on the surface of collagen bundles which have different acoustic properties from the surrounding ground substance and the cells. ${ }^{12}$ Influx of water into the dermis causes separation and disarrangement of collagen bundles and consequently a drop in echogenicity. Ultrasound method seems to be useful in clinical practice and in research for measurement of skin oedema. It has been proposed to estimate the degree of inflammatory skin reactions, particularly those evoked by patch testing, by ultrasound-based measurement of the drop in echogenicity. ${ }^{3-6}$ Ultrasonography may be suitable for measurement of skin oedema in limbs with deep venous insufficiency which is of direct clinical importance in relation to monitoring the efficacy of compressive therapy in patients with venous leg ulcers. ${ }^{10}$ Echogenicity of dermis ${ }^{8}$ and other organs such as the liver, changes in a circadian rhythm. 
probably reflecting physiological diurnal variations in water content in the tissues. In all these studies, however, the rationale for use of ultrasonography for skin water measurements has been based entirely on theoretical assumptions and no validation studies have been performed. ${ }^{1} \mathrm{H}$ nuclear magnetic resonance (NMR) is recognized as a specific method for non-invasive and quantitative determination of water in biological materials. ${ }^{13-17}$ The ${ }^{1} \mathrm{H}$ spectrum contains two major peaks derived from water and fat. ${ }^{14-16}$ Therefore, assuming that the fat content in a given anatomical site in the skin is constant, water may be determined by the ratio of the intensity of water and fat signals. ${ }^{15,16}$ Water content can also be quantified from NMR images. ${ }^{15,18}$ However, this method is cumbersome and considered less reliable than spectroscopy, as calibration against phantoms is necessary and time of scanning is significantly prolonged with a risk of movement artefacts.

In this study we evaluate if the echogenicity of the dermis is correlated to dermal water expressed as the water/fat ratio (W/F) calculated from ${ }^{1} \mathrm{H}$ magnetic resonance skin spectra.

\section{Methods}

Subjects

Fifty-nine healthy volunteers (29 men, 30 women, age 18-65, median 36) entered the study after informed consent. Ethical approval for the study was obtained from the Ethics Committee of Copenhagen Municipality.

\section{Skin ultrasonography}

Cross-sectional, B-mode scans of the skin were obtained with a $20 \mathrm{MHz}$ ultrasound scanner Dermascan C (Cortex Technology, Hadsund, Denmark). This instrument has been described, in detail, previously. ${ }^{7-10}$ During image acquisition the gain/compensation curve was adjusted in the horizontal position at $22 \mathrm{~dB}$ as at this setting maximal A-scan peaks were obtained by scanning the standard rubber phantom (Cortex Technology). The velocity of ultrasound in the skin is $1580 \mathrm{~m} / \mathrm{s}$. To obtain a uniform image size, and to ensure that images were obtained from the same site, the studied area was marked with an adhesive ring (Beiersdorf, Germany). The ultrasound gel (Greenscan, Nicomed, Denmark) was applied to the aperture of the ring and any excess was removed. The ultrasound probe was gently placed over the ring, with the transducer directed perpendicularly to the surface of the skin. Ultrasound images were recorded on floppy discs and further processed by image analysis (GIPS, Image House, Copenhagen, Denmark). This system measures echogenicity of single image elements (pixels) on a numerical scale extending from 0 (non-echogenic) to 255 (maximal echogenicity).

In each image we counted the number of pixels of intensities 0-30 (low echogenic pixels-LEPs). This method has been used previously to quantify the lowechogenic component of the ultrasound image. ${ }^{3-11}$ The number of LEPs increases with the decrease of echogenicity. LEPs were determined in the dermal region, between the epidermal entrance echo and the interface with the subcutaneous fat layer. To measure skin thickness, simultaneous A- and B-mode scans of the skin were obtained and the peaks in the A-scan corresponding to the epidermal entrance echo and the interface between the skin and the subcutaneous fat were identified. The distance between these two peaks was the measure of the thickness of the skin and equalled $1 \cdot 34 \pm($ mean \pm standard deviation: SD $0.19) \mathrm{mm}$ on the dorsal site of the forearm, $1.16 \pm 0.17 \mathrm{~mm}$ on the ventral site, increasing to $1.56 \pm 0.21 \mathrm{~mm}$ within the histamine weal. All ultrasound examinations were run in duplicate and their means were used for subsequent analyses.

\section{NMR investigations}

Proton-1 ( $\left.{ }^{1} \mathrm{H}\right)$ NMR spectroscopy was performed in a Vivospec $^{\mathrm{TM}}$ spectrometer (Otsuka Electronics, Inc., U.S.A.) equipped with a 2.9 Tesla horizontal superconducting magnet $(26 \mathrm{~cm}$ bore, $80 \mathrm{~cm}$ long) (Magnex Scientific Ltd, U.K.). The surface probe was a meander coil inductively driven and tuned for ${ }^{1} \mathrm{H} .{ }^{19}$ The ${ }^{1} \mathrm{H}$ spectra were obtained by a signal-pulse technique. Data were collected in $2 \mathrm{~K}$ memory over $205 \mathrm{~ms}$ and 10 scans were summed with a $6 \mathrm{~s}$ interpulse delay. In order to measure the depth of the penetration of the probe, a Petri dish filled with a 5-mm slap of water was placed at different distances over the surface coil. After acquisition of the spectra the area under the waterspecific peak was calculated by a curve-fitting routine (Otsuka Electronics) and plotted vs. the distance between the coil and the water slap. The area under the water-specific peak sharply decreased over the distance of $1.5 \mathrm{~mm}$ attaining $25 \%$ of the initial value at $2 \mathrm{~mm}$ from the coil.

To acquire magnetic resonance spectra (MRS) in vivo the subjects put their forearms into the magnet so that 


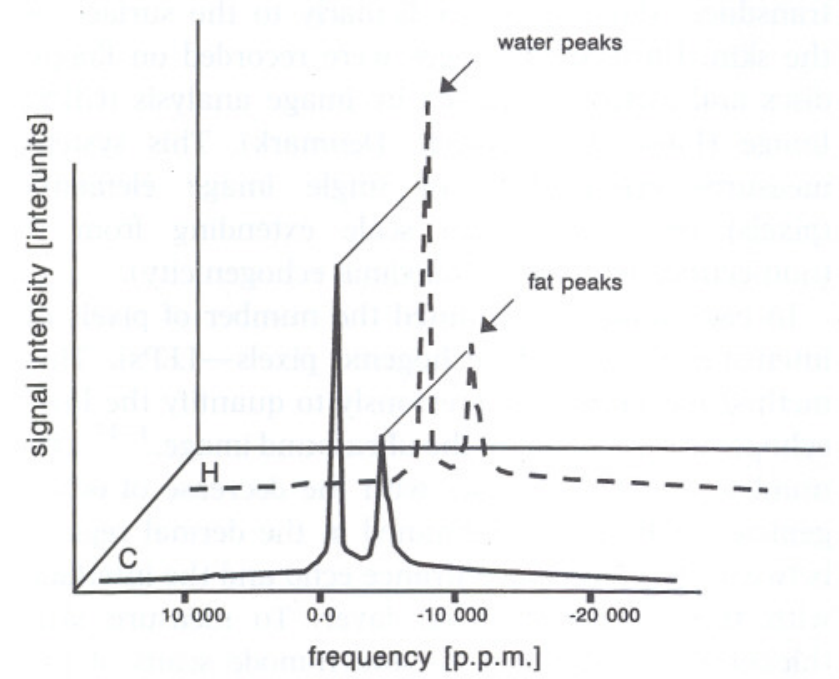

Figure 1. Magnetic resonance spectra (MRS) of human skin in vivo. Typical MRS of the control skin $(\mathrm{C})$ and after injection of histamine $(\mathrm{H})$ are shown. Water- and fat-specific peaks are indicated. Note the increase of amplitude of the water-specific peak after application of histamine.

the investigated region of the skin marked with the adhesive ring rested directly over the surface coil. Typical spectra contained two major peaks, representing water and fat (Fig. 1). The areas under the water and fat peaks were determined with the curve-fitting software. The relative amount of water in the skin was calculated by dividing the area under the water-specific peak by the area of the fat-specific peak $(\mathrm{W} / \mathrm{F})$. The advantage of using $\mathrm{W} / \mathrm{F}$ is that the relative intensity variation of individual components of the ratio, e.g. due to coil position, are eliminated. Fat is a relatively stable component of the skin and there are no major age-related changes in fat content. ${ }^{20}$

The skin imaging module consisted of a gradient surface coil placed inside and in the middle of a main magnet. The field of view was $40 \mathrm{~mm}$ and the thickness of the slice was $1 \mathrm{~mm}$. Spin-to-relaxation time (TR) was $500 \mathrm{~ms}$ and time of echo (TE) was $40 \mathrm{~ms}$. Initially, we performed chemical selective imaging where two complementary images were obtained: one related to the mobile water fraction and the other to the lipid fraction. ${ }^{21}$ To obtain better visualization of structural elements water and fat images were subsequently mixed at a standard ratio $2: 1$.

\section{Study design}

The sites on the ventral and dorsal side of the left forearm, one-third the distance from wrist to elbow, were marked with an adhesive ring (Beiersdorf). The ultrasound scan of the demarcated skin was obtained, and MRS of the same skin region was performed immediately after. Subsequently, in 40 individuals, a histamine weal was produced within the marked skin on the ventral side, as described previously. ${ }^{22}$ The standard prick test needles (Phazet, Pharmacia, Uppsala, Sweden) were used, which puncture the skin to a standard depth (mean \pm SD) of $0.79 \pm 0.05 \mathrm{~mm}$. Ten minutes after histamine application, ultrasonography followed by MRS were performed. In selected cases, ultrasound and NMR imaging of the skin were performed before and after application of histamine. Skin over the medial malleolus was chosen for this purpose, because the length of the imaging coil did not allow studies of the skin on upper extremities.

\section{Statistical analysis}

Data are presented as means with $95 \%$ confidence intervals. The $\mathrm{W} / \mathrm{F}$ data did not show a normal distribution and a logarithmic transformation was performed to normalize variations. Correlation analysis was performed to evaluate the relation between LEP number and W/F. To assess the influence of age and sex on the relationship between $\mathrm{W} / \mathrm{F}$ and LEP a multiple regression analysis was performed. Partial correlation coefficients and their confidence intervals were calculated from published formulae. ${ }^{23}$ The agreement between the ultrasound and MRS methods was assessed by calculating the $95 \%$ agreement intervals, as proposed by Bland and Altman. ${ }^{24}$ In the experiment with the histamine weal the proportional increases in LEP and W/F ratio were determined, and the differences between these values were calculated. The $95 \%$ confidence intervals of the difference between LEP and $\mathrm{W} / \mathrm{F}$ were referred to as limits of agreement. Narrow limits of agreement, when compared with the mean of the measured value, indicate good agreement between the methods. $P<0.05$ was considered statistically significant. All calculations were performed with the Minitab statistical software (Minitab, Inc., State College, U.S.A.).

\section{Results}

To measure whether baseline echogenicity of the dermis is correlated with the relative water content in the skin, LEPs were counted in the dermal ultrasound image and their number plotted against the $\mathrm{W} / \mathrm{F}$ ratio of the same region (Fig. 2). The correlation coefficients attained 
dorf). The obtained, performed ividuals, a arked skin sly. ${ }^{22}$ The harmacia, e the skin $0.05 \mathrm{~mm}$ ultrasonon selected skin were istamine. for this g coil did ities.

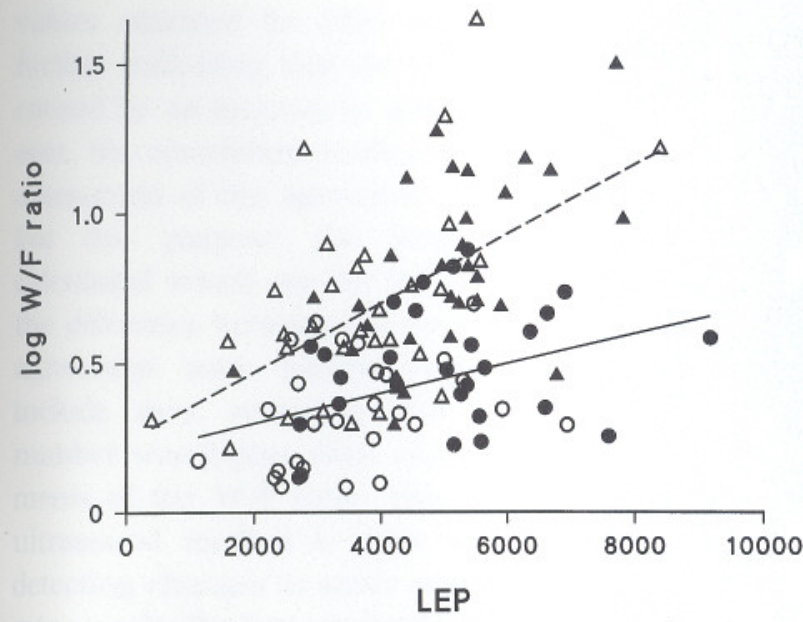

Figure 2. Relationship between the water- and fat-specific (W/F) ratio and low echogenic pixel (LEP) number of the skin. LEP were determined from the ultrasound images of the skin and $W / F$ representing relative water content was calculated from magnetic resonance spectra, as described in Materials and Methods. The logarithmic transformation of $\mathrm{W} / \mathrm{F}$ data was performed to normalize variations. Circles and triangles represent values obtained from women and men, respectively. Open symbols: ventral forearm, solid symbols: dorsal forearm. Solid line shows a significant linear correlation $(r=0.56, P<0.001)$ in male subpopulation, collectively for the data from ventral and dorsal sites. The corresponding line for the female subpopulation is plotted with a dashed line $(r=0.42$. $P<0.001)$.

statistical significance both for the ventral and dorsal site and equalled $0.42 \quad(95 \%$ confidence intervals $0 \cdot 16-0 \cdot 59)$ and $0.31(0 \cdot 05-0 \cdot 52)$, respectively. In preliminary experiments with NMR imaging we noticed that women had a thicker and more compact fat layer than men. We hypothesized that sex-linked differences in fat distribution and content might constitute a confounding factor influence of which could explain wide confidence intervals for regression coefficients. Age could be another variable affecting W/F. Therefore, we constructed a multiple regression model where $\mathrm{W} / \mathrm{F}$ ratio (after logarithmic transformation) was regressed against three predictors: the number of LEPs, sex and age. For both ventral and dorsal sites, we obtained statistically significant regression coefficients for LEPs $(P<0.0001)$ and sex $(P<0.0001)$, but not for age. The $R^{2}$ parameter (adjusted) equalled $45 \cdot 2 \%$. This indicates that $\mathrm{W} / \mathrm{F}$ depends not only on LEPs but also on sex, men having higher $\mathrm{W} / \mathrm{F}$ ratio than women. To account for this fact we calculated partial correlation coefficients (adjusted for sex) for the relationship between $\mathrm{W} / \mathrm{F}$ and LEP, which equalled: $0 \cdot 47(0 \cdot 21-0 \cdot 62)$ for the ventral site, $0.57(0.30-0.68)$ for the dorsal site, and 0.49 (0.30-0.59) cumulatively for ventral and dorsal sites.

After an intradermal application of histamine the intradermal oedema in the region of the weal was imaged with NMR (Fig. 3). In the corresponding ultrasound scan the intradermal oedema was imaged as a low-echogenic region in the dermis. In the histamine weals number of LEPs increased significantly by 2110 (95\% confidence intervals 1032-3458, $P<0.001)$ pixels and this was accompanied by correlated and significant increase in $\mathrm{W} / \mathrm{F}$ ratio by a median value of $0 \cdot 26(0 \cdot 10-0 \cdot 51, P<0 \cdot 005)$.

To calculate the agreement between the ultrasound and MRS method, proportional changes in LEPs and W/F after injection of histamine were calculated. In the histamine weals LEPs increased by $63 \%(51 \cdot 3-74 \cdot 7)$ and $\mathrm{W} / \mathrm{F}$ by $8.9 \%(2.9-14.9)$. The proportional increase in LEPs was correlated with the percentage of $\mathrm{W} / \mathrm{F}$ increase (correlation coefficient of 0.39 (0.06$0 \cdot 61), P=0.01)$. To determine the limits of agreement, the $95 \%$ confidence intervals of the difference between proportional changes in LEPs and $\mathrm{W} / \mathrm{F}$ were determined. The limits of agreement between the ultrasound and NMR methods were $43 \cdot 1-63 \cdot 5 \%$ (mean $54 \cdot 1 \%$ ).

\section{Discussion}

We have documented a correlation between echogenicity quantitated from high-frequency ultrasound skin images and $\mathrm{W} / \mathrm{F}$ ratio measuring relative skin water content. This suggests that LEP count in the dermal ultrasound image depends on the concentration of water in the skin. However, although the correlation coefficient was significantly different from zero, its confidence intervals were wide. This suggests that the association between $\mathrm{W} / \mathrm{F}$ and LEPs can explain only a fraction (approximately $45 \%$ ) of the variability of our data. ${ }^{23}$ The rest can be attributed to the influence of confounding factors, including inherent deficiencies in both MRS and ultrasound methods. The MRS method, although considered to be the most specific of the currently available techniques for water quantitation in vivo, cannot serve as a gold standard. MRS spectra originate from a thin layer of skin positioned closely to the probe rather than selectively from the dermis, as in ultrasound investigations. Thus, there is a possibility of contamination of MRS spectra with signals originating from outside the dermis. Therefore, we performed a probe penetration test which revealed that most ( $75 \%)$ of the signal was gathered from a distance of 0 

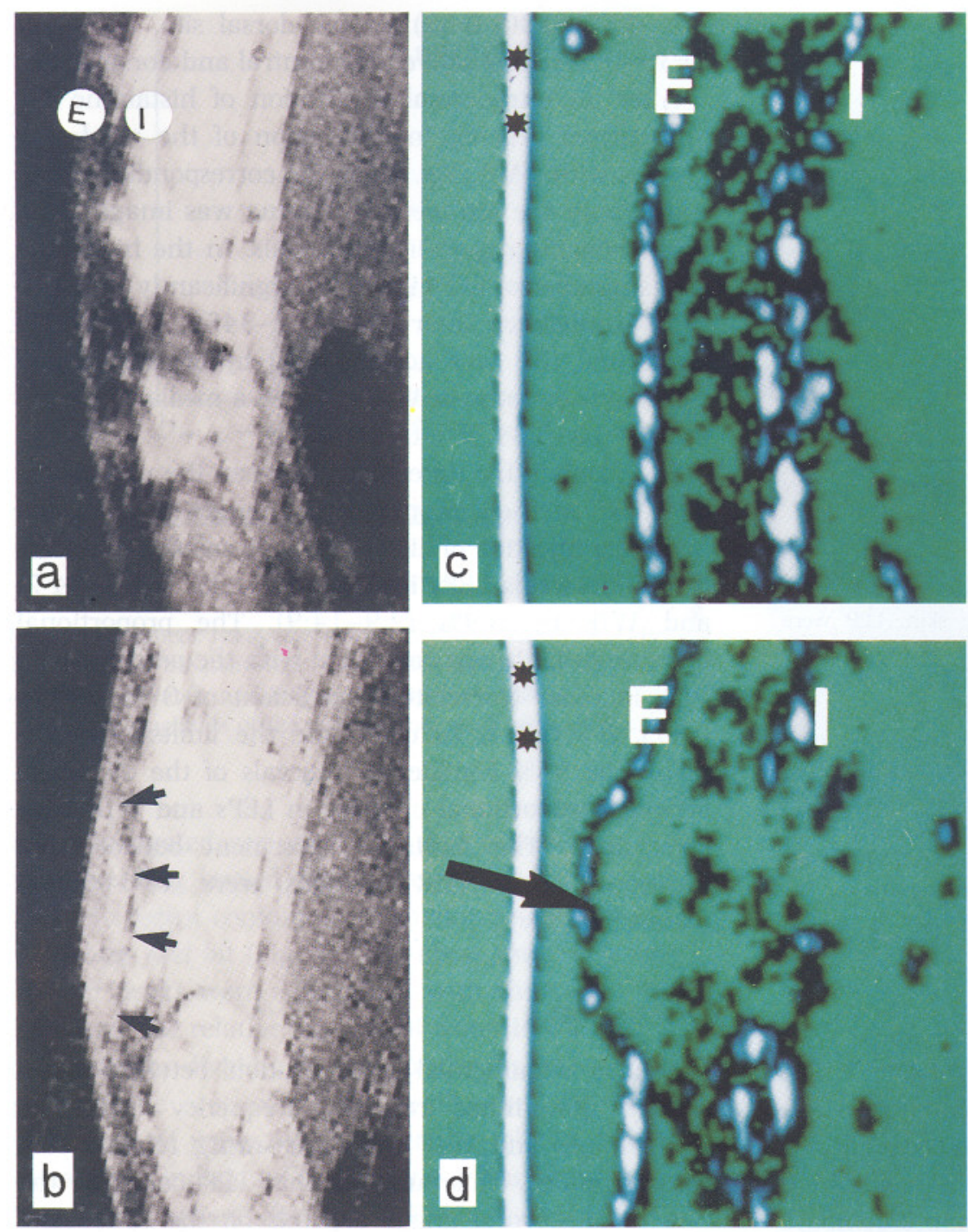

Figure 3. NMR and ultrasound imaging of human skin before and after injection of histamine. NMR image before and after histamine are shown on (a) and (b), respectively. Corresponding ultrasound images shown on (c) and (d). E-epidermis, I-interface between dermis and the subcutaneous tissue, asterisks - membrane of the ultrasound probe. Low echogenic pixels (LEPs) are highlighted in green on (c) and (d). Note a formation of a histamine weal, which presents as a water-rich structure on (b) and a hypoechogenic (LEPenriched) area on (d) (arrows).

to $1.5 \mathrm{~mm}$ from the surface of the probe, which matched observed skin thickness. Spectral components derived from the deeper layers of the subcutaneous and muscular tissues, although having low intensities, could constitute a confounding factor in the measurements. Moreover, we have shown that sex-linked differences in subcutaneous fat distribution and content influenced $\mathrm{W} / \mathrm{F}$ and, thus, modulated a relationship between W/F and LEP number (Fig. 2).

The deficiency of the ultrasound method is that the changes in echogenicity are not entirely specific for alterations in skin hydration. For example, changes in the orientation of collagen fibres may affect echogenicity. Scars and keloids present as hypoechogenic areas on the ultrasound images. ${ }^{12}$ In cutaneous malignancies, such as malignant melanoma and basal cell carcinoma, the dermal tissue becomes echolucent due to the erosion of the collagen fibre network. ${ }^{25}$ It is thus, possible that the interindividual variability in collagen architecture caused by, e.g. skin ageing and photodamage, could influence LEP counts without affecting skin water content.

To assess directly the usefulness of ultrasonography for assessment of the skin water compartment, oedema of the dermis was evoked in the histamine weal experiment, and changes in LEPs and W/F were determined. Two-dimensional sectional images through the histamine weal demonstrated a region of decreased echogenicity within dermis which corresponded to oedema in NMR images. To compare quantitatively MRS and ultrasonography, the proportional increase in $\mathrm{W} / \mathrm{F}$ ratio and LEP number were calculated. The
Ackn

We th assistí the st Leo ? Fond Switi

Refi 
values obtained for LEPs and $\mathrm{W} / \mathrm{F}$ were correlated further indicating that the increase in LEPs is indeed caused by an increase in dermal water content. However, the correlation coefficient cannot be used for an assessment of the agreement between the methods. ${ }^{24}$ For this purpose, the limits of agreement were calculated which are the $95 \%$ confidence intervals of the difference between the two methods. The limits of agreement were narrow $(43 \cdot 1-63 \cdot 5)$ but did not include zero, revealing that the increase in LEP number was higher than expected from the measurements of the $W / F$ ratio. This indicates also that the ultrasound method is more sensitive than NMR in detecting changes in water concentration in the histamine weals. The low sensitivity of MRS was unexpected, but can be explained by the influence of the epidermis on MRS spectra. The epidermis is likely to emit strong resonance signals, being a well-hydrated and lipid-rich structure, ${ }^{26}$ positioned very close to the MRS surface coil. As hydration of the epidermis in a histamine weal does not increase, the proportional change in W/F could be lower than that of LEP which was measured selectively in the dermis.

In summary, the present study indicates that high resolution $20 \mathrm{MHz}$ ultrasonography is a useful and sensitive tool for determination of water in the skin. This method cannot be used for direct and specific measurements of water but seems to be adequate for comparative evaluations where changes in water content are investigated. Being relatively inexpensive and portable, the ultrasound method could become of major importance for the in vivo study of clinical conditions with dermal oedema.

\section{Acknowledgments}

We thank Ib Therkelsen for an excellent technical assistance and Martine Evensen for help in organizing the study. Financial support was obtained from The Leo Foundation, Ballerup, Denmark; The Haensch Fond, Denmark and Sigvaris Ganzoni \& CIE AG, Switzerland.

\section{References}

1 Aukland K, Reed RK. Interstitial-lymphatic mechanisms in the control of extracellular fluid volume. Physiol Rev 1993; 73: 1-78.

2 Mani R, Ross JN, Keefe M. Measurement of limb oedema in chronic venous disease. Skin Res Technol 1995; 1: 51-4.

3 Seidenari S, Di Nardo A. B scanning evaluation of allergic reactions with binary transformation and image analysis. Acta Derm Venereol (Stockh) 1992; 175: 3-7.
4 Seidenari S, Di Nardo A. B scanning evaluation of irritant reactions with binary transformation and image analysis. Acta Derm Venereol (Stockh) 1992; 175: 9-13.

5 Seidenari S, Di Nardo A. Cutaneous reactivity to allergens at 24-h increases from the antecubital fossa to the wrist: an echographic evaluation by means of a new image analysis system. Contact Dermatitis 1992; 26: 171-6.

6 Di Nardo A. Seidenari S. Echographic evaluation with image analysis of histamine-induced weals. Skin Pharmacol 1994; 7: 285-90.

7 Gniadecka M. Gniadecki R, Serup J, Søndergaard J. Ultrasound structure and digital image analysis of the subepidermal low echogenic band in aged human skin: diurnal changes and interindividual variability. J Invest Dermatol 1994; 102: 362-5.

8 Gniadecka M, Serup J, Søndergaard J. Age-related chànges of skin oedema by high-frequency ultrasound. Br J Dermatol 1995; 131: $849-55$.

9 Serup J, Keiding J, Fullerton A et al. High-frequency ultrasound examination of skin: introduction and guide. In: Handbood of Noninvasive Methods and the Skin (Serup J, Jemec GBE, eds). Boca Raton: CRC Press, 1995: 239-56.

10 Gniadecka M. Dermal oedema in lipodermatosclerosis: distribution, effects of posture and compressive therapy evaluated by highfrequency ultrasonography. Acta Derm Venereol (Stockh) 1995; 75: 120-4.

11 Gniadecka M, Non-invasive methods for determination of oedema and water behaviour in the skin. Skin Res Technol 1995; 1: 55-60.

12 Altmeyer P. Hoffman K. Stucker M. General phenomena of ultrasound in dermatology. In: Ultrasound in Dermatology (Altmeyer P, El-Gammal S, Hoffmann F, eds). Berlin: SpringerVerlag, 1992: 55-79.

13 Cuono CB, Armitage IM, Marquetand R, Chapo GA. Nuclear magnetic resonance spectroscopy of the skin: predictive correlates for clinical application. Plast Reconstr Surg 1988; 81: 1-11.

14 Bottomley PA. Human in vivo NMR spectroscopy in diagnostic medicine: clinical tool or research probe? Radiology 1989; 170: $1-15$.

15 Schmidt SJ, Lai HM. Use of NMR and NMI to study water relations in foods. Adv Exp Med Biol 1991; 302: 405-52.

16 Schmidt SJ. Determination of moisture content by pulsed nuclear magnetic resonance spectroscopy. Adv Exp Med Biol 1991; 302: 599-613.

17 Zemtsov A, Thian CNG, Min XUE. Human in vivo 31P spectroscopy of skin: potentially a powerful tool for non-invasive study of metabolism in cutaneous tissue. J Dermatol Surg Oncol 1989; 15: 1207-11.

18 Rajanayagam V, Fabry M, Gore JC. In vivo quantification of water content in muscle tissue by NMR imaging. Magn Reson Imaging 1991; 9: 621-5.

19 Vandenborne K, McCully K, Kakihara H. Metabolic heterogeneity in human calf muscle during maximal exercise. Proc Natl Acad Sci USA 1991; 88: 5714-18.

20 Pearce RH, Grimmer BJ. Age and chemical constitution of normal human dermis. J Invest Dermatol 1972; 58: 347-61.

21 Dixon WT. Simple proton spectroscopic imaging. Radiology 1984; 153: 189-93.

22 van Neste D. Skin response to histamine: reproducibility study of dry skin prick method and the evaluation of microvascular changes with laser Doppler flowmetry. Acta Derm Venereol (Stockh) 1991; 71: 25-8.

23 Altman DG. Practical Statistics for Medical Research. London: Chapman \& Hall, 1992.

24 Bland JM, Altman DG. Statistical methods for agreement 
between two methods of clinical measurement. Lancet 1986; i: 307-10.

25 Harland CC, Bamber JC, Guesterson BA, Mortimer PS. High frequency resolution B-scan ultrasound in the assessment of skin tumours. Br J Dermatol 1993; 128: 525-32.
26 Richard S, Querleux B, Bittoun J et al. Characterization of the skin in vivo by high resolution magnetic resonance imaging: water behaviour and age-related effects. J Invest Dermatol 1993; 100: 705-9. 